\title{
Forecasting Gold Prices in India using Time series and Deep Learning Algorithms
}

\author{
P. Sai Shankar, M. Krishna Reddy
}

\begin{abstract}
The primary object of this paper is to compare the traditional time series models with deep learning algorithm.The ARIMA model is developed to forecast Indian Gold prices using daily data for the period 2016 to 2020 obtained from World Gold Council. We fitted the ARIMA $(2,1,2)$ model which exhibited the least AIC values. In the meanwhile, MLP, CNN and LSTM models are also examined to forecast the gold prices in India. Mean absolute error, mean absolute percentage error and root mean squared errors used to evaluate the forecasting performance of the models. Hence, LSTM model superior than that of the other three models for forecasting the gold prices in India.
\end{abstract}

Keywords: Gold Prices, Box-Jenkins Methodology, ARIMA,Lag Variables, MLP, CNN and LSTMModels

\section{INTRODUCTION}

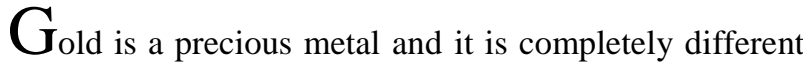
from other assets and metals. It is highly liquid and sensitive to price changes (Ranson and Wainwright, 2005). A majority of gold is bought as jewelry items. In the gold consumption, India and China together account almost 60\% of worldwide gold jewelry. Gold is consider as a traditional gift in many weddings and often given away on the occasion offestivals, such as Diwali, Akshaya Tritiya and Dantheras.Gold plays a unique role as a store of value and hedge risks (Taylor, 1998; Hammoudeh et al., 2010). People investing in gold have mainly two primary objectives, one being hedge against inflation as over a period of time (Baur and McDermott (2010) and Baur and Lucey (2010) and next is to mix your investment basket and hence diversify the risk and will help you reduce the overall volatility of your portfolio. Typically the return on gold investment is almost in line with the inflation rate. In modern days, people has to choose different ways to invest the gold by buying jewelleries which is safer way or by purchasing goldcoins and bars which is available in public sector banks nowadays or by investing in Gold Exchange traded fund (Gold ETF).Gold ETF is in financial instrument of mutual fund in nature which in turn invests in gold and these are listed in a stock index.

Manuscript received on April 24, 2021.

Revised Manuscript received on May 03, 2021.

Manuscript published on June 30, 2021.

* Correspondence Author

P Sai Shankar*, Department of Statistics, University College of Science (OU), Hyderabad (Telangana), India. Email: saishankar26@gmailcom

Dr. M. Krishna Reddy, Department of Statistic, CVR College of Engineering, and Technology, Hyderabad (Telangana), India. Email: reddymk54@gmailcom

(C) The Authors. Published by Blue Eyes Intelligence Engineering and Sciences Publication (BEIESP). This is an open access article under the CC BY-NC-ND license (http://creativecommons.org/licenses/by-nc-nd/4.0/)
Time series can be defined as a sequence of data points which is ordered in sequence and collected at regular time intervals. Time series approach can be used on any data which is changes over time. Time series are used in statistics, weather forecasting, sales forecasting, stock market predictions etc. In decisions, that involve factor of uncertainty of the future, time series models found one of the most effective methods of forecasting. The classical time series analysis procedure decomposes the time series data into four components: Trend, Seasonality, Cyclic and residual component.

There are a wide range of techniques to forecast the gold prices. There are classic econometric and statistical techniques like the exponential smoothing method (ESM), autoregressive moving averages(ARMA), auto regressive integrated moving averages (ARIMA) etc., Although these methods are useful to capture the linear relationship and they fail to capture the nonlinear characteristics of gold prices. To tackle this problem, artificial intelligence (AI) models are implemented to forecast the gold prices. The common methods are Feedforward neural networks, Multilayer perceptron (MLP), Recurrent neural networks (RNN), Convolutional neural networks (CNN) and Long short term memory (LSTM).

\section{MATERIALS AND MEHODS}

The data collected from World Gold Council of gold prices in Rupees per gram, daily frequency ranging from January 2016 to December 2020 consisting a total of 1304 observations. The train and test datasetsconsists of 1277 and 27 respectively. We built the model on train dataset and predictions on test dataset.

Table 1: Average Daily Gold Prices (Rs/gram)

\begin{tabular}{|c|c|c|c|c|}
\hline \multicolumn{5}{|c|}{ Average Daily Gold Prices (Rs/gram) in India } \\
\hline Mon & Tue & Wed & Thu & Fri \\
\hline 2755.606 & 2756.874 & 2755.702 & 2756.907 & 2755.783 \\
\hline
\end{tabular}

\section{A. Review of Box and Jenkins Approach}

The Box-Jenkins methodology is one of the well known method in the field of time series. This method applies ARIMA models to find the best fit of a time series to past values of this time series, in order to make accurate future forecasts. These methods are also commonly used when the data is notstationarity.

Let $\left\{Z_{t}\right\}$ be the time series. Then $\left\{Z_{t}\right\}$ is stationary if $E\left(Z_{t}\right)=\mu$ and $V\left(Z_{t}\right)=\sigma_{Z}^{2}$ for all $t$. Otherwise it is non -stationary. Let $Z_{1}$ ,$Z_{2} \ldots Z_{N}$ be an observed sample. If there is no trend line and constant variance for allvalues of $t$ in the time series plot, then the time series is said to be stationary. Alternatively, if the ACF of sample diesout slowly is an indication for stationary.

Published By:

Blue Eyes Intelligence Engineering and Sciences Publication

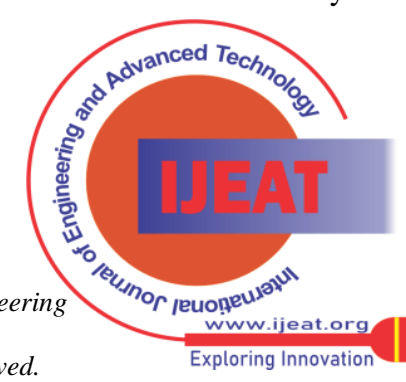


The Box - Jenkins Methodology is valid for only stationary time series data. If the data is non - stationary, we convert it into stationary by stabilizing mean using successive differencing and stabilizing variance using logarithmic transformation. The Auto Regressive Integrated Moving Average model for the time series is denoted by ARIMA(p, d, q) and is defined by $\varphi(B) \nabla^{d} Z_{t}=\theta(B) a_{t}$ where $\varphi(B)=1-$ $\varphi_{1} B-\varphi_{2} B^{2}-\cdots \varphi_{P} B^{P}$ is polynomial in $\mathrm{B}$ of order $\mathrm{p}$ and is known as Auto Regressive (AR) operator, $\theta(B)=1-\theta_{1} B-$ $\theta_{2} B^{2}-\cdots \theta_{q} B^{q}$ is a polynomial in B of order $\mathrm{q}$ and is known as Moving Average (MA), operator, $\nabla=1-\mathrm{B}, \mathrm{B}$ is the Backward shift operator $B^{k} Z_{t}=Z_{t-k}$ and d is the number of differences required to arrive stationarity timeseries. $\operatorname{AR}(p), \operatorname{MA}(q)$ and $\operatorname{ARMA}(p, q)$ may be obtained as particular case of it with parameter values $(p, 0,0),(0,0, q)$ and $(p, 0, q)$ respectively.

The four steps procedure forBox-Jenkins method: Identification of model, Parameter estimation, diagnostic checking and finally model forecast.

\section{B. Review on Deep Learning Algorithms}

In the study of ANN, the most popular algorithm is a feed-forward neural network (FFNN). It has only forward connections in between the neurons. ANNS are commonly used for time series forecasting. ANNs can model any form of unknown relationship in the data with few assumptions. ANNmodels trained on train dataset and it can generalise on unseen data. Traditional time series are capable of modelling only liner relationships where as ANNs are capable of modelling any form of relationship in the data, especially non-linear relationships (Hornik et al., 1989). The forecasting accuracies are also better than other traditional time series models.

Tang et al. (1991) compared ARIMAmodel with deep learning model for forecasting and results show that deep learning models are better than the ARIMA. Claveria and Torra (2014) studiedperformance of ANNs and traditional time series ARIMA and finally showed that ANNs are higher accuratethan ARIMA model to a tourism-demand forecasting problem. Zhang \& Kline (2007) paper revealed that the accuracy of the model is depends on the input variables, best network structure and also training procedure.

\section{Multilayer Perceptron (MLP)}

A multilayer perceptron(MLP)is a class of feedforwad artificial neural network(ANN). MLP Neural Networks can be formed a relation between the input and output data and it can be modelled by neural networks (Bildirici et al., 2010).It is an extension of feed forward neural network. A multilayer perceptron neural network with two hidden layers is depicted in Fig. 1 which consists of three types of layersthe first one input layer and output layer these two connected with hidden layers. In MLP, the input nodes are connected to the output nodes with the help of hidden layers in between the two layers. The input will be changed under the effect of the hidden layers, which behave in nonlinear way and the results of the changes multiply the weights will be transferred to the output nodes.Unlike to a feed forward network in a MLP the data flows in the forward direction from input to output layer. The number of neurons are trained with the back propagation learning algorithm. The major use cases of MLP are forecasting, recognition, prediction, pattern classification, and approximation.

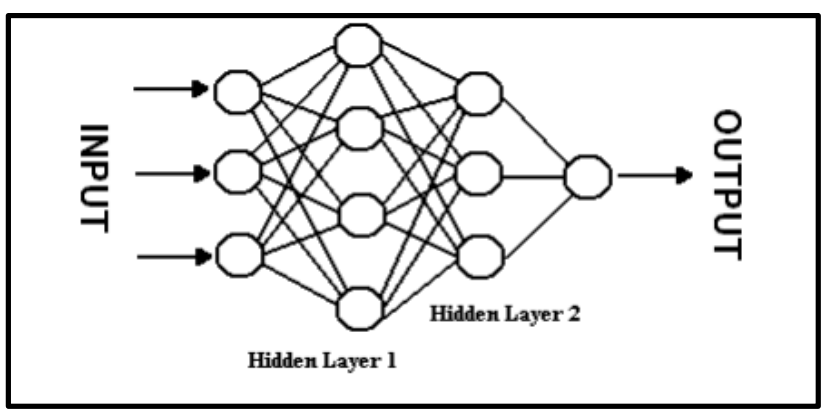

Figure 1. A simple schema of a three layer perceptron neural network

Fig. 1 representing a network structure of MLPwhere neural network has three inputs and two hidden layers with four and three nodes and one output node (3-4-3-1). A simple single layer perceptron is illustrated in the form of equation in Eq. (1).

$$
o=f\left(\sum_{i=1}^{n} w_{i} \varnothing(x)\right)(1)
$$

In Eq. (1), the variable, $x$, is the input vector, $\varnothing$ is called the activation function and the output result of the MLP could be a number or a vector of zero numbers. The variable, $w$,is the weights and $\mathrm{f}$ is the output function. Logsigmoid, ReLUand hyperbolic tangent are the different activation functions, which can be found in the neural networks literature. Activation function is the core of any node in the hidden layers of MLP changes the input of the node to the output. ReLUactivation function is used in this research which is presented in Eq. (2)

In deep learning models, the most commonly used activation function is Rectified Linear Unit. The function returns for any positive value $\mathrm{x}$ it returns that value back and 0 if it receives any negative input. It can be written as $f(x)=\max (0, x)(2)$

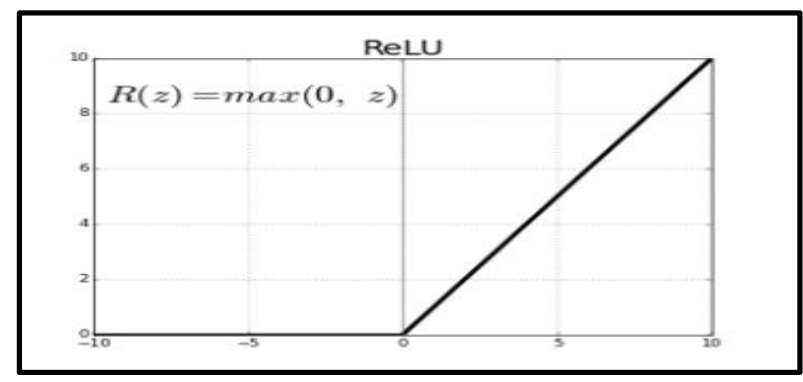

Figure 2. ReLU activation function

\section{Long Short Term Memory (LSTM)}

RNNs are a most powerful type of artificial neural network that can internally maintain memory of the input. In Recurent Neural networks(RNN) studies, one of the most common algorithm is LSTM.

The LSTM network is first developed by Hochreiter \& Schmidhuber. Since RNN has maintain memory gate, this enables them particularly used for solving the time series problems.

Blue Eyes Intelligence Engineering and Sciences Publication

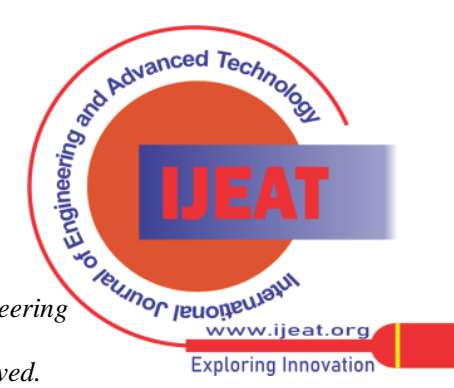


One of the drawback of RNNs isto suffer from a problem called vanishing gradient which leads to the model learning becoming too slow or stopping altogether. LSTM models are developed such that To avoid the vanishing gradient problem. LSTMs have longer memories and can learn from inputs that are separated from each other by long time lags. An LSTM has three gates: an input gate which determines whether or not to let the new input in, an output gate which decides what information to output and finally the import gate which is a forget gate which deletes information that is not useful. These three gates are analog gates based on the sigmoid function which works on the range 0 to 1 which is shown in Fig. 3 below.LSTM component is a end-to-end feature (enclosed in green dash box) with a three-layer model (consisted of input layer, LSTM unit layer and output layer).

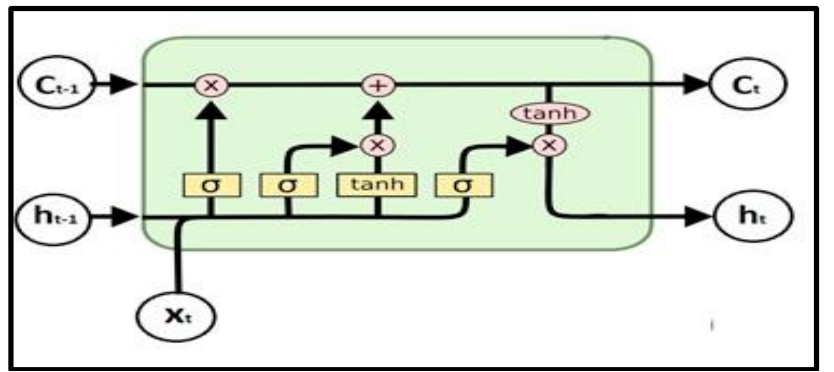

Figure 3. A simple LSTM Architect

\section{Convolution Neural Networks (CNN)}

In deep learning, a CNN is a class of Deep learning neural network, most commonly used for vision and image processing-based classification problems (object detection ,image classification, image segmentation, etc.). CNN are regularized versions of MLP. In MLP, each neuron in one layer connected with all then neurons of next layer. The "full connectivity" of these networks make them prone overfitting of the data. Regularization is one way to deal the overfitting in the data. CNN take a different approach towards regularization.

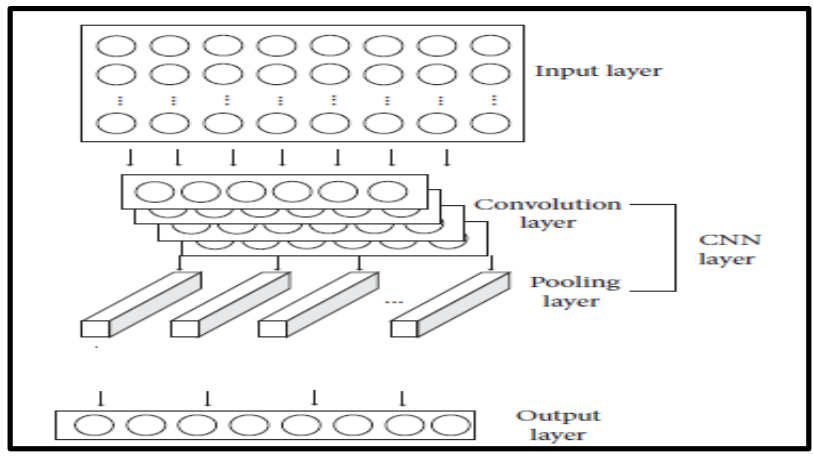

Fig 4. A simple CNN Architecture

The backpropagation process has been used to train the model in CNN model, The tuning parameters of the CNN:

- Number of hidden layers

- Number of neurons in each layer

- Learning rate

- Activation functions: ReLu, Sigmoid

- Epochs,
Batch size, Optimization algorithms: are SGD, ADAM and RMSProp

\section{Measures:}

If $Z_{t}$ is the actual price for period $t$ and $\widehat{z_{t}}$ is the forecast, then the error is defined as $\mathrm{e}=\mathrm{Z}_{\mathrm{t}}-\widehat{z_{t}}$. The following measures may be considered

Root Mean Square Error (RMSE) $=\sqrt{\frac{1}{n} \sum_{t=1}^{n} e_{t}^{2}}$

Mean Absolute Percent Error (MAPE) $=\frac{1}{n} \sum_{t=1}^{n} \frac{\left|e_{t}\right|}{z_{t}} \times 100$

Mean Absolute Error $(\mathrm{MAE})=\frac{1}{n} \sum_{t=1}^{n}\left|e_{t}\right|$

\section{ARIMA MODEL}

\section{Identification of Model}

To fit any traditional time series model, the initial step is to check the stationarity condition. Stationarity can be determined from a time series plot which should show constant mean and variance. It can also be checked from an ACF plot. Specifically, very slow decay in the lags in ACF plots indicates a non-stationarity.

\section{Testing Stationary of Time Series}

The stationary condition can also be determined by the testcalled Augmented Dickey Fuller (ADF) unit root test. The hypothesis of the test are:

The null Hypothesis $\quad \mathrm{H}_{0}$ : Xt is non - Stationary and Alternative hypothesis $\mathrm{H}_{1}$ : $\mathrm{Xt}$ is Stationary

The p-value of the Augmented Dickey-Fuller (ADF) test equals0.996 and it is larger than the value of alpha= 0.05. This result indicates that the time series of daily Gold Prices in India is not stationary.

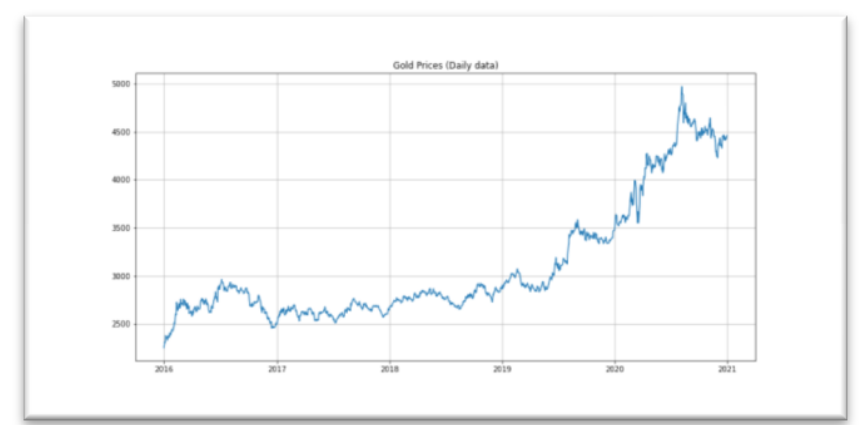

Figure 5: Time series plot of daily Gold Prices in India

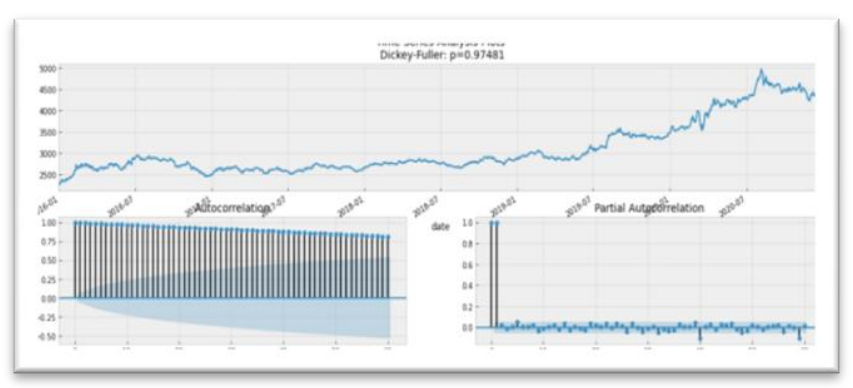

Figure 6: ACF and PACF for daily Gold Prices in India

Blue Eyes Intelligence Engineering and Sciences Publication

(C) Copyright: All rights reserved.

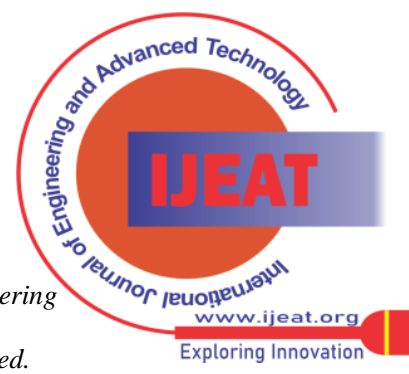


All the above plots and results confirm that the original time series data is non stationary, and need to apply some transformations to convert it into the stationary series. The differencing method is used to convert non-stationarytime series into stationary time seriesof the data. In this paper, non seasonal difference of order 1 (i.e. $d=1$ ) is sufficient to achieve stationary in mean and variance. The derived variable $. \mathrm{W}_{\mathrm{t}}=\nabla^{1} \hat{\mathrm{Z}}_{\mathrm{t}}$ can now be examined for stationary.

Figure (7) displays the time series plot of the data after first differencing the series and indicates that the time series is a stationary series. To make sure of that, we conduct the unit roots test (Augmented Dickey-Fuller) for the transformed series $\mathrm{Zt}$.

The p-value of the ADF test equals 0.00 which is less than the value of alpha=0.05 and this indicates that the nonstationary hypotheses of the differenced daily Gold Prices data is rejected and this demonstrates the success of difference transformation for the time series data of daily Gold Prices data. Thus, the series became stationary.

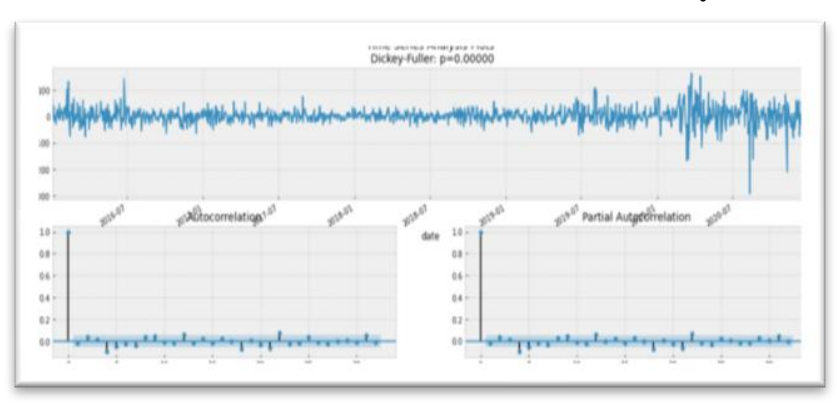

Figure 7: Time series plot,ACF and PACF of the first differences of daily Gold Prices in Indiaof the first differences of Daily Gold Prices in India.

\section{Model Identification}

This section shows how we determine the optimum ARIMA model and identify the model specifications. We computed all relevant criteria by trial and error method to select the best ARIMA model for the data. ACF and PACFare used to determine the $p$ and $q$ values. The AR(P) model is taken from the PACF plot and MA(q) model is drawn from the PACF.

From figure (7), the ACF starts from p1 value, this means that the series may be Auto Regressive (AR) and as we can observe the ACF cuts off after lag (4).On the same lines, it can be seen that the PACF of the stationary series cuts off after time lag (4).

The following tentative models have been examined and estimated as shown in table (2) below. The best ARIMA model is chosen through the AIC criteria if it shows the lowest values of these criteria.

Table (2): Tentative ARIMA Models Criteria for the daily Gold Prices in India.

\begin{tabular}{|c|c|c|c|c|c|c|c|c|}
\hline S.No & Parameters & AIC & S.No & Parameters & AIC & S.No & Parameters & AIC \\
\hline 1 & $(2,1,2)$ & $12,359.2$ & 6 & $(2,2,1)$ & $12,370.9$ & 11 & $(4,2,2)$ & $12,374.3$ \\
\hline 2 & $(4,2,1)$ & $12,361.7$ & 7 & $(1,2,1)$ & $12,371.2$ & 12 & $(1,1,2)$ & $12,374.8$ \\
\hline 3 & $(4,1,1)$ & $12,364.5$ & 8 & $(3,2,2)$ & $12,372.5$ & 13 & $(2,2,2)$ & $12,374.9$ \\
\hline 4 & $(4,1,2)$ & $12,366.4$ & 9 & $(3,2,1)$ & $12,372.8$ & 14 & $(3,1,1)$ & $12,375.0$ \\
\hline 5 & $(3,1,2)$ & $12,368.1$ & 10 & $(1,2,2)$ & $12,373.0$ & 15 & $(2,1,1)$ & $12,375.4$ \\
\hline
\end{tabular}

It is shown in table (2) that the ARIMA (2, 1, 2)is significant with respect to parameters as well as adequacy of the model. This means that the $\operatorname{ARIMA}(2,1,2)$ model is superior among all the other models.

\section{Parameters Estimation:}

\begin{tabular}{|l|c|}
\hline Dep. Variable: & Price \\
\hline Model: & ARIMA $(2,1,2)$ \\
\hline Sample: & 01-01-2016 - 24-11-2021 \\
\hline No. Observations: & 1277 \\
\hline AIC & 12359.2 \\
\hline BIC & 12384.9 \\
\hline HQIC & 12368.9 \\
\hline
\end{tabular}

Table: 3 Model Parameters of the ARIMA (2, 1, 2)

\begin{tabular}{|c|c|c|c|c|c|c|}
\hline & coef & $\begin{array}{c}\text { std } \\
\text { err }\end{array}$ & $\mathbf{z}$ & $\begin{array}{c}\mathbf{P}>\mid \mathbf{z} \\
-\end{array}$ & {$[\mathbf{0 . 0 2 5}$} & $\mathbf{0 . 9 7 5}]$ \\
\hline ar.L1 & -0.5181 & 0.015 & 33.997 & 0 & -0.548 & -0.488 \\
\hline ar.L2 & -0.9352 & 0.019 & 49.252 & 0 & -0.972 & -0.898 \\
\hline ma.L1 & 0.4994 & 0.012 & 41.209 & 0 & 0.476 & 0.523 \\
\hline ma.L2 & 0.9742 & 0.013 & 72.402 & 0 & 0.948 & 1.001 \\
\hline sigma2 & 935.98 & 16.118 & 58.069 & 0 & 904.38 & 967.57 \\
\hline
\end{tabular}

The fitted ARIMA model for the daily gold price in India is $\left(1+0.52 B+0.93 B^{2}\right) y_{t}=\left(1-0.50 B-0.97 B^{2}\right) e_{t}$

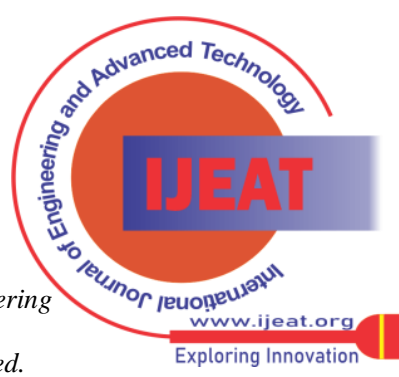




\section{Diagnostic Tests:}

The autocorrelations and partial auto correlations of the residuals plots are used for diagnostic checking.

\section{Analysis of Residuals}

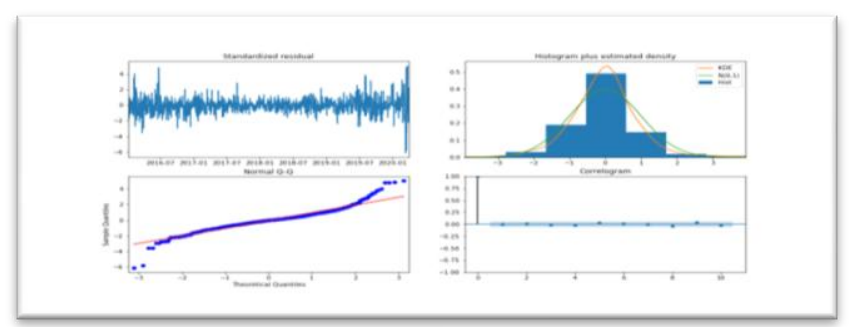

Figure (8): Residual Chart

Figure (8) show the estimated autocorrelation function for the residuals of theARIMA $(2,1,2)$ model for the time series of daily Gold prices.

Box-Ljung Q- test statistic is used to test the adequacy of the model. The hypothesis on the model is below:

HO: The ARIMA $(2,1,2)$ model is adequate.

H1: The model is $\operatorname{ARIMA}(2,1,2)$ inadequate.

Table (4): Ljung-Box Test Statistic

\begin{tabular}{|l|l|l|l|r|}
\hline Ljung-Box (Q): & 0.06 & & Jarque-Bera (JB): & 7091.2 \\
\hline Prob(Q): & 0.81 & & Prob(JB): & 0 \\
\hline Heteroskedasticity (H): & 3.21 & & Skew: & -0.67 \\
\hline Prob(H) (two-sided): & 0.00 & & Kurtosis: & 14.47 \\
\hline
\end{tabular}

From the Table(4),the probability value of the test statistic is 0.81 which is greater than 0.05 , therefore, we fails to reject Ho. Hence, we conclude that the ARIMA $(2,1,2)$ model is an adequate model for the giventime series data.

\section{DEEP LEARNING MODELS}

\section{A. Multilayer Perceptron (MLP)}

Python software has been used to train the MLP model on evaluate the model on test dataset.

\section{Structure of the Network:}

The MLP model consists of three: input layer, a hidden layer and an output layer. Two input neurons considered in this model, each representing the values of lag1 (previous day price in the same week i.e. lag1 gold price) and lag2 (lag 2 gold price). One output unit is needed in this modelwhich indicates the forecasts of daily gold price. Trial and error approach can be usedto find the optimal number of hidden units in the neural network. Typically we can use either the forward selection method or backward selectionto arrive the optimumthe hidden layer units. In the forward selection method, we choose a small number of hidden neurons then compute the network performance using RMSE, MAE and MAPE values. In the next step increment the hidden neuronsby one until train and test error is acceptably small or no further improvement is noted.

\begin{tabular}{|c|c|c|}
\hline Layer (type) & Output Shape & Param \# \\
\hline 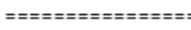 & 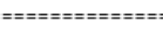 & $:=======3$ \\
\hline dense (Dense) & (None, 500) & 1500 \\
\hline dense_1 (Dense) & (None, 500) & 250500 \\
\hline dense_2 (Dense) & (None, 1) & 501 \\
\hline \multicolumn{3}{|c|}{ 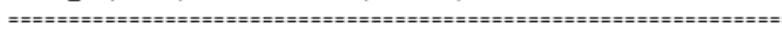 } \\
\hline \multicolumn{3}{|c|}{ Total params: 252,501} \\
\hline \multicolumn{3}{|c|}{ Trainable params: 252,501} \\
\hline \multicolumn{3}{|c|}{ Non-trainable params: $\theta$} \\
\hline
\end{tabular}

\section{B. Long Short-Term Memory (LSTM)}

Python software has been used to train the LSTM model on evaluate the model on test dataset.

\section{Structure of the Network:}

Model: "sequential_1"

\begin{tabular}{|c|c|c|}
\hline Layer (type) & Output Shape & Param \# \\
\hline \multicolumn{3}{|c|}{ 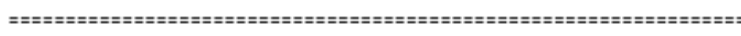 } \\
\hline lstm (LSTM) & (None, 1000) & 4008000 \\
\hline dense_4 (Dense) & (None, 1) & 1001 \\
\hline \multicolumn{3}{|c|}{ 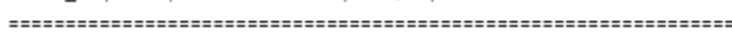 } \\
\hline \multicolumn{3}{|c|}{ Total params: 4,009,001 } \\
\hline \multicolumn{3}{|c|}{ Trainable params: $4,009,001$} \\
\hline \multicolumn{3}{|c|}{ Non-trainable params: $\theta$} \\
\hline
\end{tabular}

\section{Convolutional neural networks (CNNs)}

Python software has been used to train the CNNmodel on evaluate the model on test dataset.

\section{Structure of the Network:}

\begin{tabular}{|c|c|c|}
\hline Layer (type) & Output Shape & Param \# \\
\hline 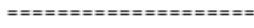 & 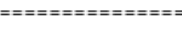 & $==x===s$ \\
\hline conv1d_6 (Conv1D) & (None, 1, 64) & 192 \\
\hline flatten_5 (Flatten) & (None, 64) & $\theta$ \\
\hline dense_17 (Dense) & (None, 500) & 32500 \\
\hline dense_18 (Dense) & (None, 1) & 501 \\
\hline \multicolumn{3}{|c|}{ 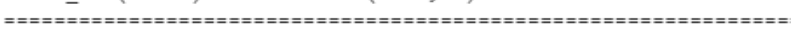 } \\
\hline \multicolumn{3}{|c|}{ Total params: 33,193} \\
\hline \multicolumn{3}{|c|}{ Trainable params: 33,193} \\
\hline Non-trainable params & & \\
\hline
\end{tabular}

\section{RESULTS AND DISCUSSION}

The train dataset is used to train the models. Several traditional and deep learning models ARIMA, MLP, CNN and LSTM are examined.Once we trained the dataset using training dataset then we test the model on test dataset. The performance measures are showed in the Table5. Figures 912 represents the line charts of actual gold price and predicted gold prices.

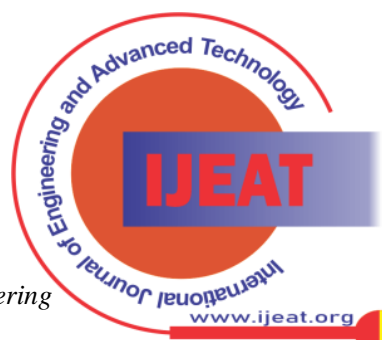


Table 5: Accuracy measures for different models to predict daily gold price (Rs/gram) in India

\begin{tabular}{|c|c|c|c|}
\hline Forecasting Model & Error Measures & Train Set & Test set \\
\hline \multirow{4}{*}{ ARIMA } & MAPE & 0.7 & 2.19 \\
\cline { 2 - 4 } & RMSE & 70.1 & 110.55 \\
\cline { 2 - 4 } & MAE & 21.68 & 97.07 \\
\hline \multirow{4}{*}{ MLP } & MAPE & 0.69 & 0.72 \\
\cline { 2 - 4 } & RMSE & 33.28 & 38.85 \\
\cline { 2 - 4 } & MAE & 22.13 & 31.21 \\
\hline \multirow{4}{*}{ CNN } & MAPE & 0.71 & 0.74 \\
\cline { 2 - 4 } & RMSE & 33.95 & 39.93 \\
\cline { 2 - 4 } & MAE & 22.66 & 32.23 \\
\hline \multirow{3}{*}{ LSTM } & MAPE & 0.65 & 0.69 \\
\cline { 2 - 4 } & RMSE & 31.46 & 35.87 \\
\cline { 2 - 4 } & MAE & 20.59 & 29.31 \\
\hline
\end{tabular}

The experimental results revealed that the LSTM model is the best among the four methods. In terms of forecasting performance measures, MAPE is 0.69, MAE is 29.31 and RMSE is 35.87, which is the lowest among the four forecasting models. Therefore, the LSTM model is superior to the other three comparative models in terms of forecasting gold prices in India.

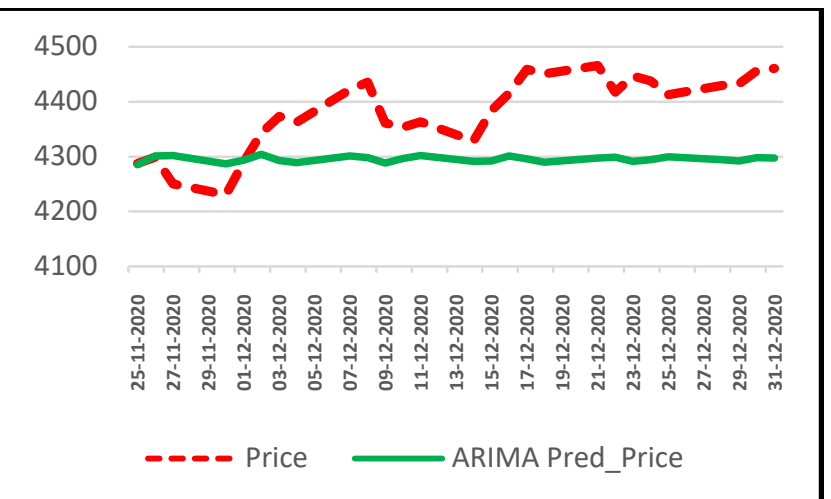

Figure. 9: ARIMA prediction (forecasted) of gold price(Rs/gram).

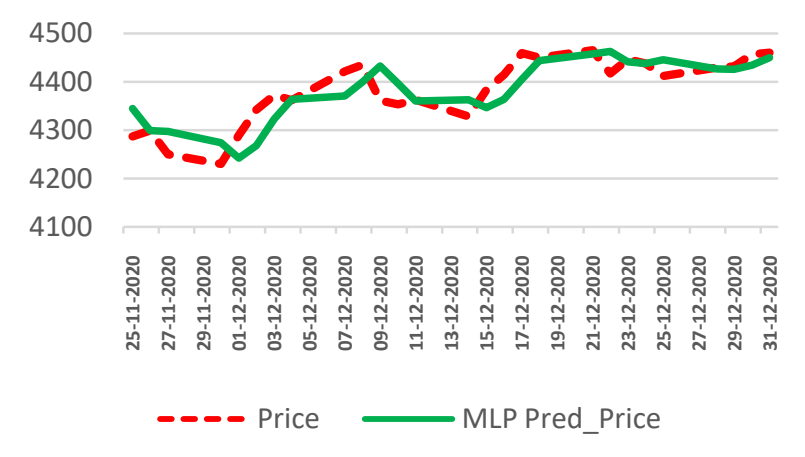

Figure. 10: MLP prediction (forecasted) of gold price(Rs/gram).

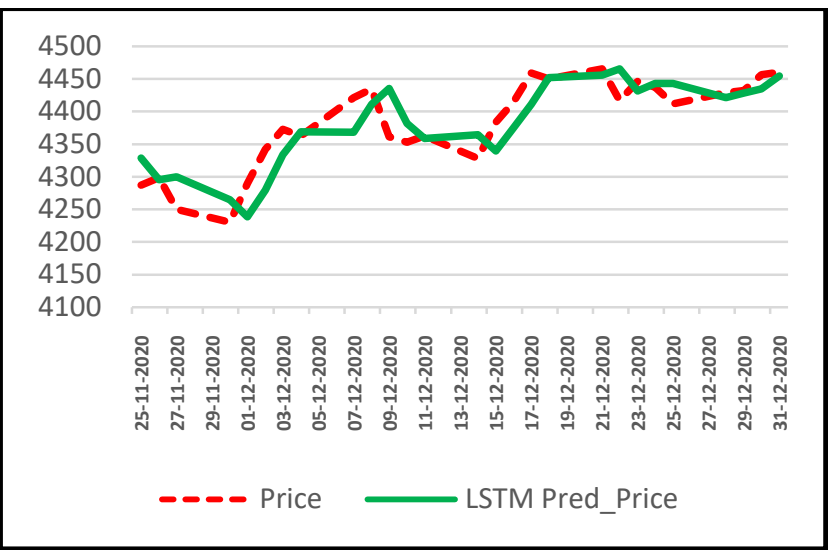

Figure 11: LSTM prediction (forecasted) of gold price(Rs/gram).

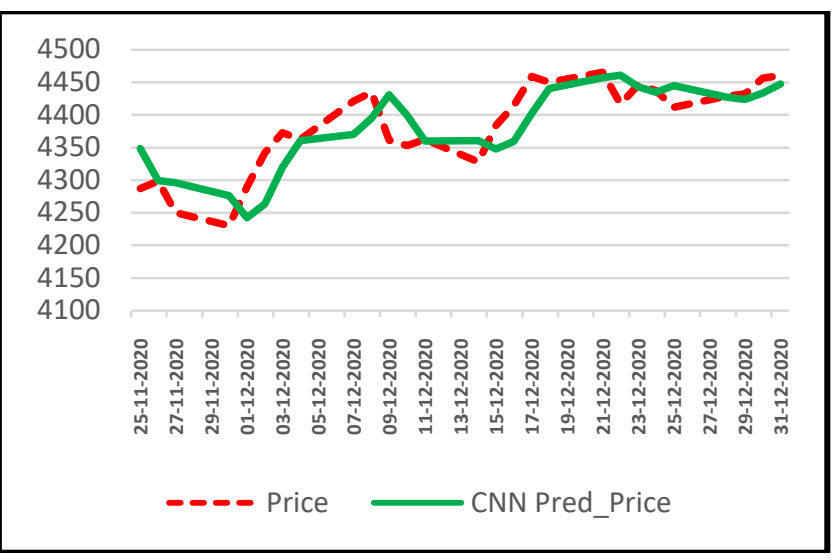

Figure. 12: CNN prediction (forecasted) of gold price(Rs/gram).

\section{CONCLUSIONS}

The main object of the paper is to compare the traditional time series models with deep learning algorithm. The historical data is collected from World Gold Council of gold prices in Rupees per gram, daily frequency ranging from January 2016 to December 2020. LSTM model outforms than other three modelsto forecast the gold price in India using historical data. This study is based on secondary data collected from. The analysis showed that the LSTM model has the lowestMAPE, RMSE and MAE and performed better compared with the traditional time series model like ARIMA, MLP and CNN.However, the model can be improved further by incorporate other factors such as US dollar, Crude oil, Inflation and Bank rates into the forecastmodel. Our future research work ismainly to incorporate some explanatory variables which are influences the gold prices to ensure the accuracy of gold forecast.Forecasts for test data using LSTM model presented in the following table (6).

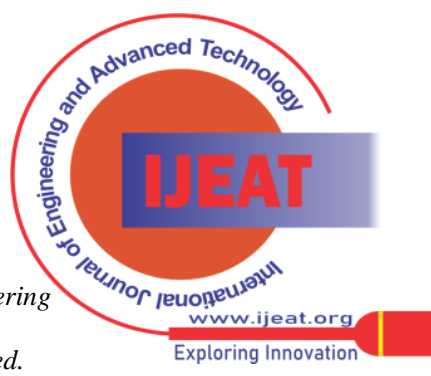


Table 6. Gold price forecasts using LSTM model for the test dataset

\begin{tabular}{|c|l|l|l|l|l|}
\hline Date & Price & Pred_Price & Date & Price & Pred_Price \\
\hline $25-11-20$ & 4286.8 & 4328.7 & $15-12-20$ & 4383.6 & 4339.7 \\
\hline $26-11-20$ & 4298.6 & 4295.0 & $16-12-20$ & 4414.2 & 4374.3 \\
\hline $27-11-20$ & 4249.6 & 4299.6 & $17-12-20$ & 4458.8 & 4410.9 \\
\hline $30-11-20$ & 4230.0 & 4264.7 & $18-12-20$ & 4450.0 & 4452.2 \\
\hline $01-12-20$ & 4289.8 & 4238.2 & $21-12-20$ & 4465.3 & 4455.9 \\
\hline $02-12-20$ & 4342.1 & 4279.5 & $22-12-20$ & 4416.7 & 4465.5 \\
\hline $03-12-20$ & 4372.4 & 4333.6 & $23-12-20$ & 4446.3 & 4431.8 \\
\hline $04-12-20$ & 4362.2 & 4369.1 & $24-12-20$ & 4437.4 & 4443.2 \\
\hline $07-12-20$ & 4421.0 & 4368.3 & $25-12-20$ & 4411.9 & 4443.3 \\
\hline $08-12-20$ & 4434.9 & 4411.0 & $28-12-20$ & 4428.8 & 4421.6 \\
\hline $09-12-20$ & 4360.8 & 4435.4 & $29-12-20$ & 4431.8 & 4428.6 \\
\hline $10-12-20$ & 4352.9 & 4381.8 & $30-12-20$ & 4456.3 & 4434.9 \\
\hline $11-12-20$ & 4362.6 & 4358.5 & $31-12-20$ & 4460.0 & 4454.4 \\
\hline $14-12-20$ & 4327.8 & 4364.1 & & & \\
\hline
\end{tabular}

\section{REFERENCES}

1. Graves, A. Mohamed, and G. Hinton, "Speech recognition with deeprecurrent neural networks," in 2013 IEEE International Conference on Acoustics, Speech and Signal Processing, May 2013, pp. 6645-6649.

2. Abhay Kumar Agarwal (2020), Swati Kumari, Gold price prediction using Machine Learning, International Journal of Trend in Scientific Research and Development.

3. Anderson, J. A., (1995), "Introduction to Neural Networks", Cambridge, MA: MIT Press.

4. Amalendu Bhunial and Somnath Mukhuti (2013), "The impact of domestic gold price on stock price indices-An empirical study of Indian stock exchanges", Universal Journal of Marketing and Business Research (ISSN: 2315-5000) Vol. 2(2) pp. 035-043, May, 2013.

5. Baur, D. G., \& McDermott, T. K. (2010). Is gold a safe haven? International evidence. Journal of Banking and Finance, 34, 18861898.

6. Baur, D. G., \& Lucey, B. M. (2010). Is gold a hedge or a safe haven? An analysis of stocks,bonds and gold. The Financial Review, 45, 217229.

7. Batten, J.A., Ciner, C. and Lucey, B.M. (2010), The macroeconomic determinants of volatility in precious metals markets. Resources Policy. 35, pp.65-71

8. Box, G. E. P., Jenkins, G. M. And Reinsel, G. C (1994), "Time Series Analysis Forecasting and Control", 3rd ed.,Englewood Cliffs, N. J. Prentice Hall.

9. Brown, R. G (1959), Statistical forecasting for inventory control. New York: McGraw Hill.

10. G. Mohan Naidu*, B. Ravindra Reddy and B. Ramana Murthy (2018), TIME SERIES FORECASTING USING ARIMA AND NEURAL NETWORK APPROACHES, Int. J. Agricult. Stat. Sci. Vol. 14, No. 1, pp. 275-278, 2018

11. Hammoudeh, S., Yuan, Y., McAleer, M., Thompson, M. (2010), Precious metals-exchange rate volatility transmission and hedging strategies. International Review of Economics and Finance, 19(4), 698-710.

12. Hassan A. N. Hejase and Ali H. Assi (2012), "Time-Series Regression Model for Prediction of Mean Daily Global Solar Radiation in AlAin, UAE”, International Scholarly Research Network.

13. Iftikhar ul Sami, Khurum Nazir Junejo (2017)," Predicting Future Gold Rates using Machine Learning Approach", International Journal of Advanced Computer Science and Applications

14. Jyothi Manoj* and Suresh K K (2019), Forecast Model for Price of Gold: Multiple Linear Regression with Principal Component Analysis, Thailand Statistician

15. Matheus Henrique Dal Molin Ribeiro, Leandro dos Santos Coelho (2020), "Ensemble approach based on bagging, boosting and stacking for short-term prediction in agribusiness time series", Applied Soft Computing Journal

16. Omer Berat Sezer, Mehmet Ugur Gudelek, Ahmet Murat Ozbayoglu (2020), "Financial time series forecasting with deep learning : A

Published By:

Blue Eyes Intelligence Engineering and Sciences Publication

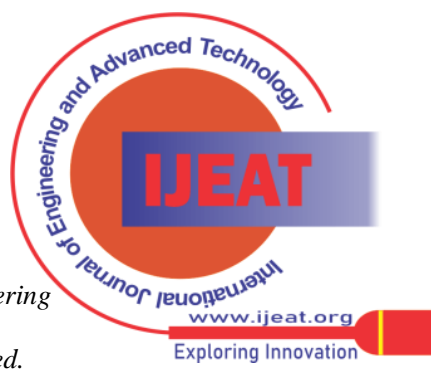

